\title{
Hybrid photosensitive structures based on nematic liquid crystals and lithium niobate substrates
}

https://doi.org/10.1515/odps-2018-0003

Received July 15, 2018; revised October 15, 2018; accepted October 23, 2018

\begin{abstract}
Liquid crystal cells based on lithium niobate substrates have recently been proposed as good candidates for optofluidic devices and for light-induced controlled generation of defects in liquid crystal films. The peculiarity of these structures lies in the possibility of using the bulk photovoltaic effect of lithium niobate to obtain an optically induced dc field able to affect the molecular liquid crystal director. Reversible fragmentation and selfassembling of liquid crystal droplets driven by the lithium niobate pyroelectric properties have also been reported. We review the basic results obtained so far with the aim of making the point and seeing what else can be done in the framework of the realization of hybrid structures combining lithium niobate with the electro-optical and nonlinear optical properties of liquid crystals.
\end{abstract}

\section{Introduction}

Lithium niobate $\left(\mathrm{LiNbO}_{3}\right)$ has been among the most widely used artificial materials in photonics [1]. The continuing popularity of $\mathrm{LiNbO}_{3}$ in this area originates from its highly desirable properties, such as large electro-optic, acousto-optic, piezoelectric, and nonlinear optical coefficients, and also the important fact that this material can be rather easily produced in a single-domain state [2, 3]. Liquid crystals (LC), on their side, are known for their ready response to external stimuli, among which optical and electric fields are widely used in photonics and display applications $[4,5]$.

\footnotetext{
*Corresponding Author: Liana Lucchetti: Dipartimento SIMAU, Università Politecnica delle Marche, Ancona, Italy, E-mail: l.lucchetti@univpm.it

Victor Reshetnyak: Physics Faculty, Taras Shevchenko National University of Kyiv, Volodymyrska street, 64, Kyiv, Ukraine
}

Recent studies [6-9] have demonstrated the possibility of combining these two materials thus taking advantage of the peculiarities of both. In particular, lightinduced control of the optical phase shift by a LC cell having iron doped $\mathrm{LiNbO}_{3}$ crystals $\left(\mathrm{LiNbO}_{3}: \mathrm{Fe}\right)$ as substrates $[6,7,10]$ and the generation and manipulation of defects in LC films deposited on $\mathrm{LiNbO}_{3}$ :Fe crystals $[8,9]$ have been reported. In all these papers the key role is played by the bulk photovoltaic effect of $\mathrm{LiNbO}_{3}$, particularly strong in iron doped crystals, able to convert optical fields into electric fields [11]. The bulk photovoltaic effect is manifested by the appearance of a photo-induced current, which is generated in $\mathrm{LiNbO}_{3}$ upon illumination. The subsequent charge redistribution that takes place inside the crystal gives rise to an electric field with values that can reach $10^{5} \mathrm{~V} / \mathrm{cm}$. The electric field associated with the photovoltaic current is referred to as photovoltaic field [11]. Thanks to this peculiar effect, the combination of liquid crystals and lithium niobate paves the way to the realization of novel microfluidic and photonic solutions that mix the peculiarities of the different constituents.

In addition, previous studies have shown that the high surface electric fields due to $\mathrm{LiNbO}_{3}$ pyroelectricity lead to reversible fragmentation and self-assembling of nematic liquid crystal droplets [12], thus including the pyroelectric effect among the lithium niobate properties that can be successfully combined with the LC responsiveness.

In this review the basic results obtained so far on $\mathrm{LC} / \mathrm{LiNbO}_{3}$ hybrid structures are recalled with the aim of making the point and investigating further possible configurations.

\section{Light-induced control of the optical phase shift in $\mathrm{LC} / \mathrm{LiNbO}_{3}$ structures}

The first experiment on a $\mathrm{LC}$ cell with $\mathrm{LiNbO}_{3}$ substrates was reported by Evans et al. in 2006 [13]. With a very simple 
experimental set-up consisting of a cw green laser beam linearly polarized and focused on the sample, the authors demonstrated the possibility of affecting the LC molecular orientation through the photovoltaic fields induced by light in each of the two $\mathrm{LiNbO}_{3}$ substrates enclosing the LC film. Specifically, they reported the photoinduced behavior of two kind of systems: a planar cell used as a spatial filter and a twisted cell used as a light valve. In the first case, the light transmitted by the cell upon light irradiation was detected as a function of time and a fast decrease followed by an increase of the signal was observed. This behavior was accounted for considering that, due to the Gaussian profile of the incident light beam, the buildup of charges and the subsequent field across the LC layer were initially strongest in the center of the Gaussian beam. For this reason LC molecules were reoriented to different degrees across the inhomogeneous region of illumination and the LC film acted as a lens to strongly diverge the incident light. When charges built up also at the less intense edges of the beam, the lensing effect disappeared thus reducing the divergence of the transmitted beam (see Fig. 1). In the second case, light transmission between crossed polarizers was measured. The induced LC reorientation destroyed the initial longitudinal twist of the cell and the incident beam did not experience a rotation in polarization as it propagated through the cell. The presence of the crossed analyzer allowed for birefringent switching, causing the cell to function as a light valve. A secondary process of power transfer was also observed due to photorefractive two-beam coupling in $\mathrm{LiNbO}_{3}$ crystals involving the incident and reflected beams, this effect being much slower than LC reorientation driven by the photovoltaic fields. This paper has the value of being the first to propose this novel kind of LC cells and to demonstrate the influence of the lithium niobate bulk photovoltaic effect on the LC orientation; however, quantitative data on the induced phase shift were reported only ten years later, when similar hybrid structures were independently proposed $[6,10]$.

In works [6, 10], LC planar cells were realized using zcut (i.e. with the c-axis normal to the surfaces) $\mathrm{LiNbO}_{3}: \mathrm{Fe}$ substrates that appear as optically isotropic when irradiated by light polarized in the plane of the cell. Good planar alignment of the LC molecules was obtained by treating the two $\mathrm{LiNbO}_{3}$ crystals with a bath of sulphuric acid and hydrogen peroxide. This made the crystals surfaces hydrophilic thus allowing the coating with Polyvinyl-alcohol, the usual surfactant used to obtain planar LC cells. Mechanical rubbing followed the PVA deposition to define the orientation of the LC molecular director. As demonstrated in those papers by pump-probe ex-

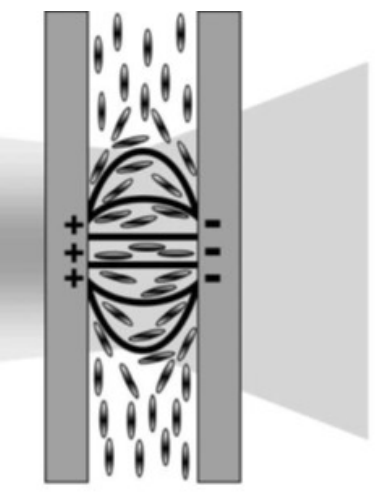

(a)

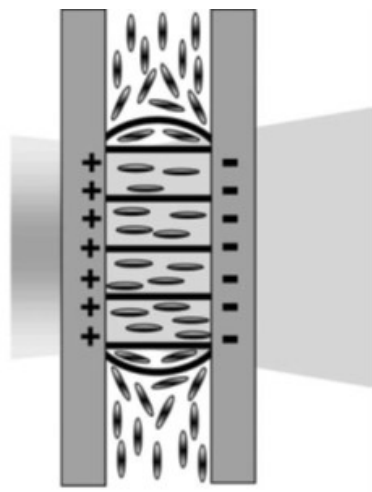

(b)
Figure 1: Sketch of a LC cell with LiNb03 substrates. The shaded region represents the portion of the cell illuminated by a laser beam propagating from left to right. (a) At the beginning of light irradiation, the Gaussian profile of the incident light results in a graded index of refraction in the LC film. (b) As a steady state is reached, surface charges gradually build up on the substrates at the less intense edges of the incident Gaussian beam, strengthening the field in this region. Reprinted with permission from ref [13], [OSA].

periments, a light-induced control of the phase shift could be easily achieved with single beam illumination, corresponding to an induced optical anisotropy $\Delta \mathrm{n}$ of about 0.1 , making negligible any contribution originated from photorefractive nonlinearities of the $\mathrm{LiNbO}_{3}: \mathrm{Fe}$ crystals. The phase shift was directly measured by detecting the probe light intensity transmitted by the cell upon pump irradiation with the pump beam polarized parallel to the unperturbed LC optic axis and the probe beam polarized at $45^{\circ}$ with respect to the pump. Specifically, the transmitted light was divided into two signals: one polarized parallel and one orthogonal to the polarization direction of the probe light incident on the sample. Each of the two signals is related to the phase shift $\Delta \varphi$ experienced by the light travelling through the cell according to the relations [14]:

$$
\begin{aligned}
& I_{\perp}=I_{0} \exp \left(-\alpha_{o} d\right) \sin ^{2} \frac{\Delta \phi}{2} \\
& I_{\|}=I_{0} \exp \left(-\alpha_{e} d\right) \cos ^{2} \frac{\Delta \phi}{2}
\end{aligned}
$$

where $\mathrm{d}$ is the LC film thickness and $\alpha_{o, e}$ are the absorption coefficients for light polarized orthogonal and parallel to the molecular director. Therefore, if the difference between the absorption coefficients is negligible, the induced phase shift can be easily determined from the ratio 
between the intensities $I_{\perp}$ and $I_{\|}$of the two beams [14]:

$$
\begin{aligned}
& \Delta \phi=N \pi+2 \tan ^{-1} \sqrt{\frac{I_{\perp}}{I_{\|}}} \text {if } N=0,2,4, \ldots \\
& \Delta \phi=(N+1) \pi+2 \tan ^{-1} \sqrt{\frac{I_{\perp}}{I_{\|}}} \text {if } N=1,3,4, \ldots
\end{aligned}
$$

where the integer $\mathrm{N}$ represents the number of extrema (maxima and minima) in the curves $\mathrm{I}_{\perp}$ and $\mathrm{I}_{\|}$.

An example of the behavior of the detected signals as a function of the exposure time is shown in Fig. 2, where $\mathrm{I}_{\perp}$ and $\mathrm{I}_{\|}$are reported in the same graph [6]. The signals show the typical oscillations observed when a planar sample, in the same geometry as the one used in the experiment, is exposed to an increasing voltage, where each additional extrema corresponds to an increase of $\pi$ of the phase shift between the two waves. The final situation corresponds to almost uniform director alignment perpendicular to the substrates, thus allowing transmission of the signal parallel to the incoming polarization. In the present case the pump intensity is fixed and the photovoltaic field in the substrates gradually increases with time, which leads to a time increasing electric field across the LC cell. It is seen that a phase shift of several $\pi$ can be easily reached.

In order to exclude possible contributions from the direct effect of light on the LC, similar measurements on conventional cells with glass substrates were performed and no response was detected. Empty $\mathrm{LiNbO}_{3}$ cells were also tested with the same result. The observed reorientation thus came from the combination of $\mathrm{LiNbO}_{3}$ with its bulk photovoltaic effect and LC with its high sensitivity to electric fields.

The observed effect was used to create a high contrast optical switch based on the transmission decrease induced by the LC transition from planar to homeotropic configuration under the action of the optically generated electric field [6]. The response time of this optical switch was measured to be on the order of $1 \mathrm{~s}$. Due to absence of power supply and wires, the described effect is very promising for the generation of hybrid $\mathrm{LiNbO}_{3} / \mathrm{LC}$ devices to be used in the frame of optofluidic applications where high degree of integration are required.

A detailed analysis of the electric field optically induced in the region between the two $\mathrm{LiNbO}_{3}$ substrates as a function of the crystals' properties (such as iron content and reduction factor), was published one year later [7]. In this paper the field values were first evaluated starting from the measured phase shift $\Delta \phi$ induced in the LC region, by pump probe experiments similar to those described in [6]. The dependence of $\Delta \phi$ on the electric field acting on the LC slab was derived from the minimization of

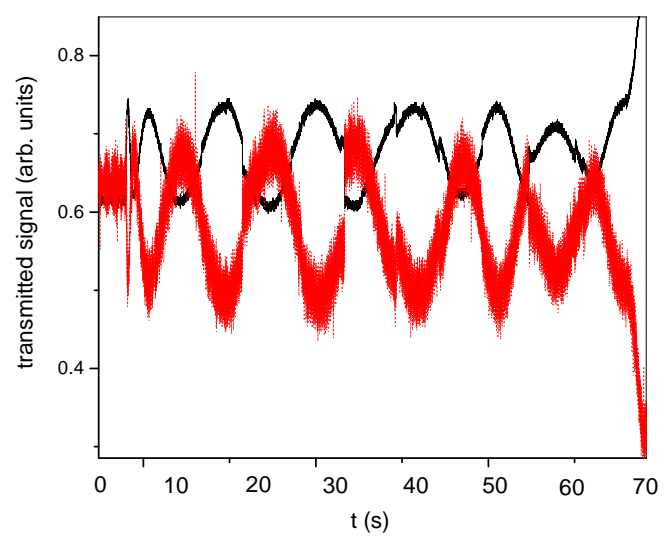

Figure 2: Transmitted probe signal with polarization parallel (dashed line) and orthogonal (solid line) to that of the probe beam incident on the hybrid LC/LiNbO3 cell, as a function of the exposure time.

the LC elastic free energy density combined with a term describing the coupling with the electric field. The expected values of the field responsible for the observed LC reorientation, reported in Fig. 3, turned out to be on the order of $(1-10) \times 10^{4} \mathrm{~V} / \mathrm{m}$.

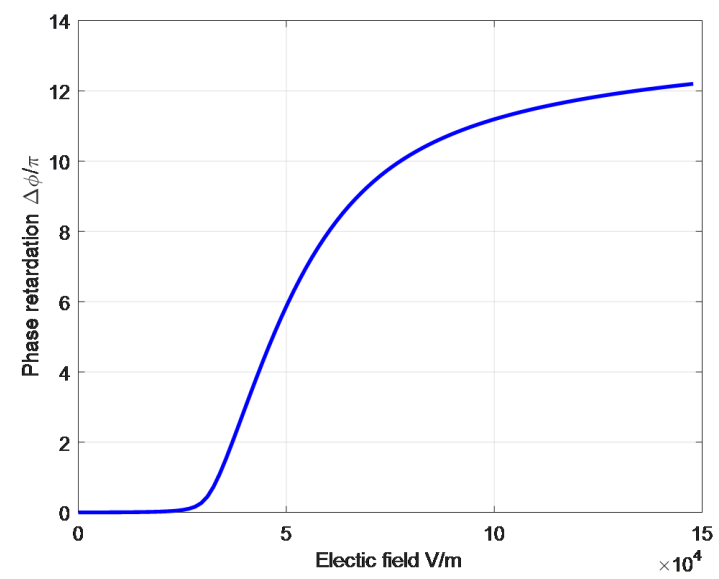

Figure 3: Phase shift experienced by the probe beam travelling through the cell, as a function of the electric field. The field is measured in $\mathrm{V} / \mathrm{m}$. Unpublished data.

The on- and off-times reported in [6] are both of the order of $1 \mathrm{~s}$. The theoretical ratio between the two is given by $\tau_{o f f} / \tau_{o n}=\frac{\varepsilon_{0} \varepsilon_{a} E^{2} d^{2}}{K \pi^{2}}$, where $d$ is the cell thickness, $K$ is the LC elastic constant, $\varepsilon_{a}$ is the LC dielectric anisotropy and $\mathrm{E}$ is the value of the field inducing the LC response. From this relation the estimate for the electric field is $6 \times 10^{4} \mathrm{~V} / \mathrm{m}$. This value is comparable to those obtained 
by measuring the photocurrent induced in the $\mathrm{LiNbO}_{3}$ irradiated area [15] and to the ones measured by methods based on the photorefractive properties of $\mathrm{LiNbO}_{3}$ crystals [16]. In Table 1 iron concentration, photovoltaic field in lithium niobate crystals and induced phase shift in the LC layer, are reported together for the illumination conditions used in the experiments described in [6-10]. It has been demonstrated that the LC reorientation induced by the photovoltaic field optically generated in the cell's substrates gives rise to substantial values of the light-induced phase shift, which paves the way to a number of interesting applications, such as all-optical devices to be integrated in platforms exploiting lithium niobate as a substrate for optofluidic circuits.

Table 1: Iron concentration, photovoltaic field in lithium niobate crystals and induced phase shift in the LC layer. Reprinted with permission from ref [7].

\begin{tabular}{cccc}
\hline$\#$ & $\mathbf{C}_{F e}$ & $\mathbf{E}\left(\star^{*} \mathbf{0}^{6} \mathbf{V} / \mathbf{m}\right)$ & $\Delta \varphi$ \\
\hline $\mathbf{1}$ & $0 \%$ & $0.002 \pm 0.001$ & 0 \\
$\mathbf{2}$ & $0.002 \%$ & $0.009 \pm 0.001$ & 0 \\
$\mathbf{3}$ & $0.01 \%$ & $1.6 \pm 0.4$ & $1.3 \pi$ \\
$\mathbf{4}$ & $0.05 \%$ & $6.2 \pm 0.4$ & $1.4 \pi$ \\
$\mathbf{5}$ & $0.1 \%$ & $10.1 \pm 0.8$ & $13.2 \pi$ \\
$\mathbf{6}$ & $1 \%$ & $0.4 \pm 0.1$ & $5.2 \pi$ \\
\hline
\end{tabular}

The following step was to model the field acting on the LC film assuming a space charge density with Gaussian distribution on the surfaces of the irradiated lithium niobate area [7]. Specifically, a surface charge density with Gaussian profile which mimics that of the incident intensity was assumed:

$$
\sigma_{\text {sufrace }}=\sigma_{0} \exp \left(-\left(\frac{r-D / 2}{w}\right)^{2}\right)
$$

being $\mathrm{w}$ the beam waist and $\mathrm{D}$ the modelling domain width $(\mathrm{D}=5 \mathrm{w}$ and $\mathrm{w}=750 \mu \mathrm{m})$. The electric field generated by these light-induced charged areas was evaluated by solving the Maxwell equations for the system of the two photovoltaic substrates with the LC layer in between. The director was fixed in the planar configuration and the calculations were done in 3D. The resulting electric field profile is reported in Fig. 4. It is seen that the field is inhomogeneous in the plane of the cell and is essentially uniform along the cell thickness. The value of the field inside the LC layer is on the order of $10^{5} \mathrm{~V} / \mathrm{m}$, which is nicely comparable to the values in Fig. 3, calculated from the measured phase shift. As a further test of the model, the LC director profile were also evaluated and compared to the experimental results.
For this purpose the condition of fixed planar alignment was relaxed and the calculation was made in 2D. This also allowed to find the minimum value of the surface charge density required to get an almost total director reorientation from planar to homeotropic configuration. A value of $\sigma_{0}$ on the order of $10^{-4} \mathrm{C} / \mathrm{m}^{2}$ is expected.

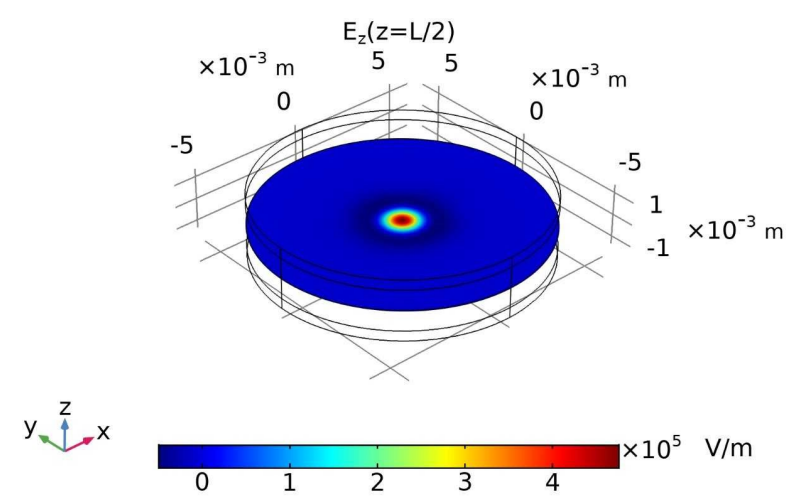

(a)

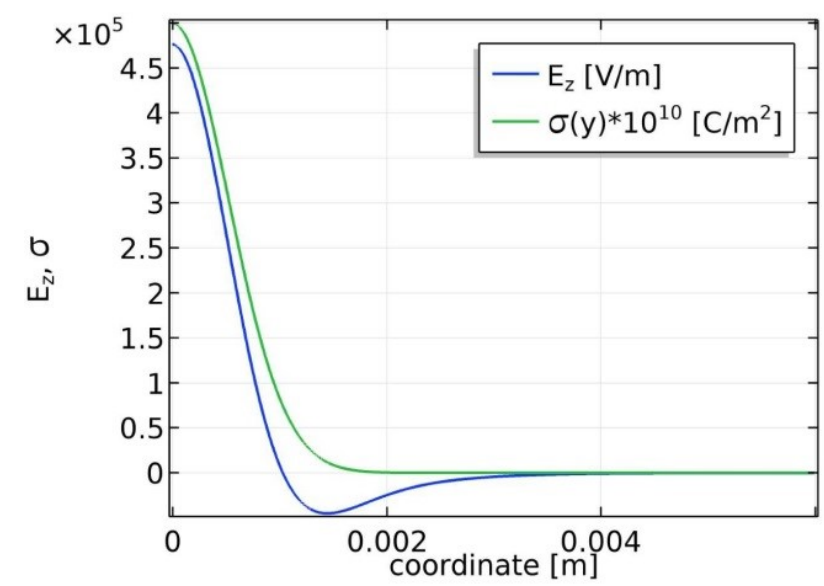

(b)

Figure 4: a) Z component of the optically induced electric field calculated as described in the text. The field is inhomogeneous in the xy plane, while it is essentially uniform along the cell thickness. Reprinted with permission from ref [7]. b) Y-dependence of the Z component of the same field. Unpublished data.

Besides the dopant concentration, another relevant parameter in determining the photovoltaic response of iron-doped lithium niobate, is the reduction degree $\mathrm{R}$ defined as the ratio between the density of $\mathrm{Fe}^{2+}$ and $\mathrm{Fe}^{3+}$ ions [16]. Even if the variation of $R$ does not critically affect the value of the photovoltaic field , it has a relevant effect on the response time of the crystals. Indeed, the characteristic time of the bulk photovoltaic effect is the dielectric 
relaxation time, having the form $[11,16]$ :

$$
\tau=\frac{\epsilon \epsilon_{0}}{\sigma_{p h}+\sigma_{d}} \cong \frac{\epsilon \epsilon_{0}}{e \mu s q} \frac{\gamma h v}{I R}
$$

where $\sigma_{p h}$ and $\sigma_{d}$ are the photo and dark conductivities, $\epsilon$ is the proper dielectric constant of $\mathrm{LiNbO}_{3}, \gamma$ is the recombination coefficient, $q$ is the quantum efficiency of excitation of an electron upon absorption of a photon, $\mu$ is the carrier mobility, $\mathrm{s}$ is the photon absorption cross section, $v$ is the frequency of the light and I is the incident intensity.

Thus, highly reduced samples are expected to exhibit a faster response, that is the induced photovoltaic field reaches the saturation value in a shorter time with respect to those with a lower reduction degree. This is expected to affect to some extent the response time of the lithium niobate based LC cells.

The role of $\mathrm{R}$ has been analyzed although results are still unpublished. The different values of the response time $t^{\star}$ of four LC cells made with $\mathrm{LiNbO}_{3}$ :Fe substrates having the same iron concentration, but different values of $\mathrm{R}$, were measured and compared (see Table 2). The response time was evaluated by pump probe experiments similar to those already described in this section, at a fixed pump intensity $\mathrm{I}=10 \mathrm{~W} / \mathrm{cm}^{2}$. It was observed that $t^{\star}$ decreases with increasing $R$, except for the case of extremely highly reduced samples. A possible explanation for this phenomenon can be the high absorption coefficient of highly reduced $\mathrm{LiNbO}_{3}: \mathrm{Fe}$ crystals. In fact, the absorption coefficient is proportional to the density of $\mathrm{Fe}^{2+}$ ions $[11,16,17]$ and, thus, it is high in crystals where $\mathrm{R}$ is high. In this case, in a LC cell based on such a type of substrates, the pump light that reaches the substrate not directly irradiated is expected to be much weaker than the light incident on the first substrate, this effect being more pronounced as $\mathrm{R}$ increases. This intensity loss, should result in a lowering of the photoconductivity and, thus, in an increase of $\tau$ (see Eq. 4), which in turn, affects the LC response time. This latter can be written as [18]:

$$
t^{\star} \cong \frac{\gamma}{\epsilon_{0}|\Delta \epsilon| E^{2}}
$$

being $\epsilon_{0}$ and $\Delta \epsilon$ the vacuum dielectric permittivity and the dielectric anisotropy of the $\mathrm{LC}$, respectively and $\mathrm{E}$ is the modulus of the electric field created in the cell by the two photovoltaic substrates. If $\mathrm{R}$ is high, the value of the photovoltaic field generated in the photovoltaic substrates under illumination, for a fixed pump intensity and a fixed irradiation time, can be low, thus giving rise to a low E field in the region filled by the LC and to a high response time $t^{\star}$.

The results reported in [7] and the discussed role of the reduction factor, demonstrate that the electric field gener-
Table 2: Iron concentration, photovoltaic field in lithium niobate crystals and induced phase shift in the LC layer. Reprinted with permission from ref [7].

\begin{tabular}{rrrllll}
\hline$\#$ & $\mathbf{C}_{F e}$ & \multicolumn{1}{c}{$\mathbf{R}$} & $\mathbf{E}\left({ }^{\star} \mathbf{1 0 ^ { 6 }} \mathbf{V} / \mathbf{m}\right)$ & $\Delta \phi$ & $\mathbf{t}^{*}(\mathbf{s})$ \\
\hline $\mathbf{1}$ & $0.1 \%$ & 0.008 & 8.0 & $<\pi$ & 300 \\
$\mathbf{2}$ & $0.1 \%$ & 0.03 & 10.06 & $13.2 \pi$ & 0.5 \\
$\mathbf{3}$ & $0.1 \%$ & 0.08 & 10.0 & $7 \pi$ & 0.1 \\
$\mathbf{4}$ & $0.1 \%$ & 1 & 7.0 & $5 \pi$ & 5 \\
\hline
\end{tabular}

ated in the LC area can be controlled and engineered by acting on the lithium niobate crystals used to build the LC cells.

\section{Defects generation in LC films through the light action on $\mathrm{LiNbO}_{3}$ crystals}

Another possible configuration of hybrid $\mathrm{LC} / \mathrm{LiNbO}_{3}$ structures, is the one combining a $\mathrm{LiNbO}_{3}$ crystal with an ordinary glass. This has been recently proposed by Habibpourmoghadam et al. to get optical formation and manipulation of defects in the liquid crystal films $[8,9]$.

In ref. [8], chiral nematic $\left(\mathrm{N}^{\star}\right)$ LC cells were obtained combining a z-cut $\mathrm{LiNbO}_{3}$ :Fe crystal and an ITO coated glass. In such a kind of LC cells the light induced electric field was observed to lead to the formation of localized point defects, that did not relax upon stopping irradiation and that could be erased by defocusing the pump beam. Experiments were performed by irradiating the $\mathrm{N}^{\star}$ cells with a focused laser beam $(\lambda=532 \mathrm{~nm})$ having a beam waist of about $15 \mu \mathrm{m}$ (the incident beam is thus much more focused than in experiments reported in [6] and [7] where the beam waist was $750 \mu \mathrm{m}$ ). The ground state configuration, obtained by mechanical rubbing of the surfaces, was characterized by uniform LC orientation with the helix axis orthogonal to cell's substrates. Because of both the use of a focused irradiating beam and the presence of only one $\mathrm{LiNbO}_{3}$ substrate, the photovoltaic field spatial distribution was found to be different with respect to the one observed in $[6,7,10,15]$, in that the in plane components of the field are now comparable to the out of plane one, as shown in Fig. 5a. In figures $5 b$ and $5 c$ the expected LC molecular director arrangement is also shown both during irradiation (red) and after removing the incident beam (green). According to the authors, within the beam waist area a focal conic state was field-stabilized, whereas outside the beam waist area, the initial alignment stays al- 
most unperturbed because the field is too weak to overcome the elastic torque. Thus, upon field-removal, the LC could relax into a defect of the Franck-Pryce kind (Fig. 5b) which can be easily erased through exposure to a defocused laser beam which generates a much smoother field distribution, as shown in Fig. 5c.

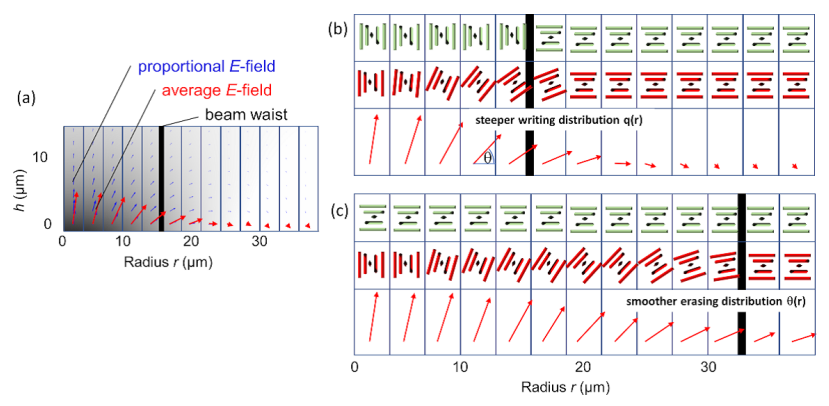

Figure 5: Field distribution, schematic of local helix realignment while field was induced (red colored helices) and in the field off state (green colored helices). The beam waist is indicated as black line. (a) Distribution of the electric field in an exposed sample, where the intensity was high enough to result in helix realignment. The average (surface near) field is shown as red arrows. For better visibility, the average field vectors (red arrows) were scaled by a factor of 2. (b) Distribution of average electric field and schematic of resulting local helix alignment in a sample exposed with a focused beam with narrow beam waist and (c) in a sample exposed with a defocused beam with broader beam waist. Reprinted with permission from ref [8], [OSA]

The described field induced point defects were used to write erasable patterns on the $\mathrm{N}^{\star} \mathrm{LC}$ film by scanning the laser beam along a programmed trajectory, thus demonstrating the high potential of the effect for reconfigurable pattern recording in $\mathrm{N}^{\star} \mathrm{LCs}$. According to authors, dye doping is not required thus samples cannot bleach, do not degrade upon exposure to non-focused light and the responses are highly reliable.

In ref. [9] experiments similar to those just described were performed on low birefringence nematic LC also. Two different configurations were tested: in the first one the LC was deposited on a $\mathrm{LiNbO}_{3}$ substrate to form an open LC film, in the second one the film was confined in the form of $30 \mu \mathrm{m}$ thick cells having one $\mathrm{LiNbO}_{3}$ crystal and one glass coverslip as substrates. In both cases all the surfaces were mechanically rubbed in order to induce planar alignment of the LC molecules. Again irradiation with a low power focused green laser beam induces the appearance of defects in the LC film. Specifically, the formation of radial and line defects was reported (see Fig. 6). The radial defects can also be entangled by the line defect, when two beams irradiation is carried out. An example of such a behavior is reported in Fig. 6c, where two radial entangled defects are clearly visible. A qualitative description of the expected induced director configuration was also proposed. An additional interesting feature of the interaction of light with the hybrid structures proposed in [9] is observed when irradiating an open LC film deposited on a photovoltaic substrate (the first configuration mentioned above) and scanning the laser beam across the edge of the film. In this way the position of the radial defect was optically moved inside the LC film and when the film edge was reached, the LC film expanded on the $\mathrm{LiNbO}_{3}$ substrate. According to authors LC expansion is due to the high polarizability of this material because of which it is attracted by dielectrophoretic forces caused by the light induced fields at the substrate surface [19]. The described edge expansion occurred in a few seconds and is shown in Fig. 7.

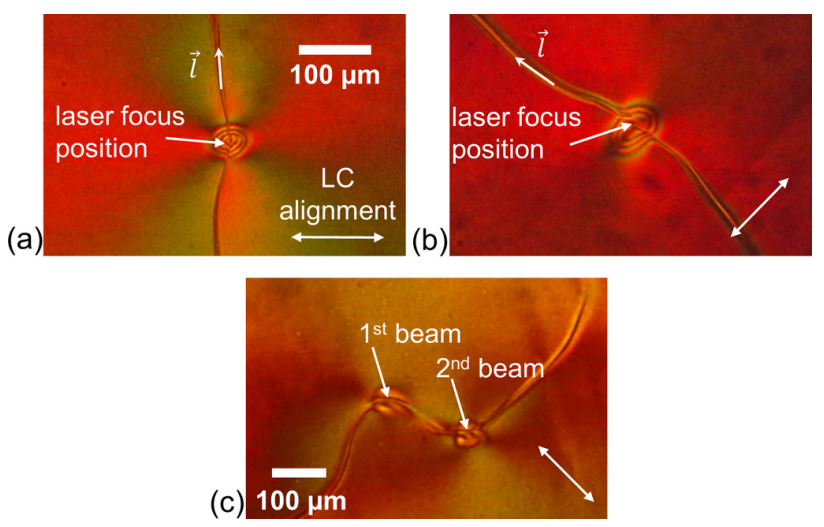

Figure 6: Defects and disclination lines in samples with a confined LC film. The alignment direction of the LC is indicated (white double arrows) and a vector $i$ is shown, which is parallel to the defect line. $(a, b)$ The sample was rotated in different positions and exposed to a single focused laser beam. (c) The sample was exposed to two adjacent focused laser beams resulting in defects connected by a merged defect line. Reprinted with permission from ref [9].
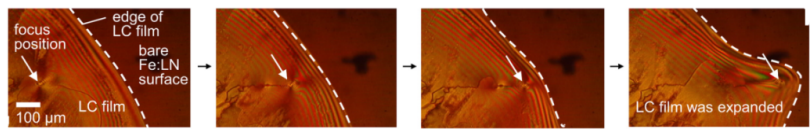

Figure 7: Sequence of images captured from a recorded video file showing the edge of the LC film. The edge of the LC film and the $\mathrm{LiNbO}_{3}: \mathrm{Fe}$ substrate surface are indicated. The focus position of the laser beam was scanned across the film edge (from left to right) within a few seconds; the film edge was thereby expanded. Adapted with permission from ref [9].

The possibility to optically generate and manipulate local structures in LC through the photovoltaic field in- 
duced by light in lithium niobate substrates, demonstrated in $[8,9]$, is clearly very promising for recording of reconfigurable patterns in view of the realization of photonic components to be used alone or integrated in optofluidic devices.

\section{Manipulation of liquid crystals through pyroelectric effect}

Dielectrophoretic forces were also proposed to explain the thermally induced phenomena occurring when the highly polar nematic LC 4-(trans-4-nhexylcyclohexyl)isothiocyanatobenzene (6CHBT) is deposited on a PDMS substrate lying on a z-cut periodically poled lithium niobate crystal [12]. Because of the hydrophobic nature of PDMS, LC arranged itself in randomly distributed droplets. An externally induced temperature variation led to the appearance of a pyroelectric field on the surface of the $\mathrm{LiNbO}_{3}$ crystal, which, due to the dielectrophoretic interaction between the induced surface charges and the strong dipole moment of the used LC, gave rise to coalescence or fragmentation of drops and migrations along the electric field lines. Subsequent studies performed on 4'-octyl-4-biphenylcarbonitrile (8CB) LC highlighted the formation of chemical bonds between the adsorbed LC and the crystal substrates that have to be taken into account besides the dielectrophoretic forces [20]. Worthy of note the effect reported in [12] is not related to light action and it could seem inappropriate to cite it in a review about photosensitive structures. However, pyroelectricity in $\mathrm{LiNbO}_{3}$ is known to arise even in case of high intensity light irradiation because of light absorption and heating [21], similar droplets manipulation can thus be expected under illumination. Results reported in [12] are thus an additional demonstration of the versatility of LC/lithium niobate combined structures and of the possibilities offered by the liaison between these two classes of materials.

\section{Conclusion}

The combination of soft matter and photoresponsive materials holds great promise in view of the realization of novel optical devices able to combine the peculiarity of the different components. Hybrid structures mixing lithium niobate and low molar mass nematic liquid crystals have recently been proposed as good candidates to be used in optofluidic devices and as promising systems for the light-induced controlled generation of defects in LC films. LC manipulation through the pyroelectric effect was also demonstrated. Results obtained so far with such combined structures have been reviewed here.

LC cells having two equal $\mathrm{LiNbO}_{3}$ substrates have been analyzed through pump probe experiments. These latter demonstrated that, due to the charge separation occurring in lithium niobate upon illumination, an electric field appears in the region in between the two substrates, which is able to affect the LC molecular orientation. Since the charge separation creating the electric field is generated by a laser beam impinging on one of the two $\mathrm{LiNbO}_{3}$ substrates, the field is locally present only in a region of the cell just slightly larger than the beam waist. A detailed study of the spatial profile of such an optically induced electric field and of the extent to which it can reorient the LC director, showed that the proposed cells are very promising for the realization of all optical devices easily integrable into optofluidic platforms, where optically induced local electric fields can readily be generated and controlled.

Open nematic LC films and cells having one $\mathrm{LiNbO}_{3}$ crystal and one glass plate as substrates have also been proposed and used for the optically controlled generation and manipulation of defects in LC. Both transient radial defects in low birefringence nematic LC and permanent, reconfigurable Franck-Pryce defects in chiral nematic LC have been observed. The possibility of locally creating field induced textural transitions in LC looks promising for reconfigurable pattern recording. Moreover, defects with radial symmetry can be used for low power optical trapping and micromanipulation of colloids, which may pave the way to the realization of light induced photonic motives such as refractive index modulations (micro lenses, resonators), and complex structures of entangled defects, which can potentially be combined with colloids and defects induced by colloids [22-26] to build reconfigurable, light induced photonic structures. Moreover, the ability to move a fluid birefringent film across a sample surface, as also demonstrated in [9] , is a technique with high potential for sensing and micromanipulation applications in microfluidic devices and lab-on-a-chip technology.

Results obtained in LC droplets systems deposited on PDMS substrates lying on periodically poled $\mathrm{LiNbO}_{3}$ crystals represents an additional novel approach for manipulating LC by means of the pyroelectric instead of the photovoltaic field [12]. The two fields may also be present together and a combination of their effects may be envisaged as an additional degree of freedom in controlling the LC response. 
Overall, the combination $\mathrm{LiNbO}_{3} / \mathrm{LC}$ has demonstrated to be fruitful and promising and several further investigations can be envisaged. For example, in all the studies reported so far $\mathrm{z}$ cut $\mathrm{LiNbO}_{3}$ crystals have been used; however studying the LC response to photovoltaic fields generated in $\mathrm{x}$ cut and $\mathrm{y}$ cut crystals alone or in combination with $\mathrm{z}$ cut ones, would be desirable, since it is known that the crystallographic orientation affects the field spatial profile [27] and new effects can be expected. The use of LC with low ionic content would also be of interest, since higher values of the induced electric fields can be expected and consequent improvements of the LC response can be foreseen. Moreover, pump probe experiments such as those described in $[6,7]$ repeated in microfluidic configuration, with the LC flowing in microchannels engraved in $\mathrm{LiNbO}_{3}$ substrates, are also very desirable in view of investigating directly the possible generation of all optical devices based on $\mathrm{LiNbO}_{3}$ optofluidic platforms. Work in this latter direction is currently in progress and results will be reported in a forthcoming paper.

\section{References}

[1] L. Arizmendi, Photonic applications of lithium niobate crystals, Phys. Status Solidi 201(2), 253-283 (2004).

[2] R. S. Weis and T. K. Gaylord, Lithium niobate: summary of physical properties and crystal structure, Appl. Phys., A Mater. Sci. Process. 37(4), 191-203 (1985).

[3] K. K. Wong, Properties of Lithium Niobate (INSPEC, 2002).

[4] F. Simoni, O. Francescangeli, Effects of light on molecular orientation of liquid crystals, Journal of Physics: Condensed Matter, 11(41), R439 (1999).

[5] Deng-Ke YangShin-Tson Wu, Fundamentals of Liquid Crystal Devices, Second Edition, 2015 John Wiley \& Sons, Ltd.

[6] L. Lucchetti, K. Kushnir, A. Zaltron, F. Simoni, Light controlled phase shifter for optofluidics, Opt. Lett., 41, 333 (2016).

[7] L. Lucchetti, K. Kushnir, V. Reshetnyak, F. Ciciulla, A. Zaltron, C. Sada, F. Simoni, Light-induced electric field generated by photovoltaic substrates investigated through liquid crystal reorientation, Optical Materials, 73, 64-69 (2017).

[8] Atefeh Habibpourmoghadam, Liana Lucchetti, Dean R. Evans, Victor Y. Reshetnyak, Faissal Omairat, Samuel L. Schafforz, and Alexander Lorenz, Laser-induced erasable patterns in a $N^{*}$ liquid crystal on an iron doped lithium niobate surface, Opt. Express, 25, 26148-26159 (2017).

[9] Atefeh Habibpourmoghadam, Lin Jiao, Victor Reshetnyak, Dean R. Evans, and Alexander Lorenz, Optical manipulation and defect creation in a liquid crystal on a photoresponsive surface, Phys. Rev. E 96, 022701 (2017).

[10] L. Lucchetti, K. Kushnir, A. Zaltron, F. Simoni, Liquid crystal cells based on photovoltaic substrates, JEOS-RP, 11, 16007 (2016).

[11] T. Volk, and M. Wohlecke, Lithium niobate (Springer-Verlag, Berlin/Heidelberg, 2008).
[12] Francesco Merola , Simonetta Grilli, Sara Coppola, Veronica Vespini , Sergio De Nicola, Pasquale Maddalena, Cosimo Carfagna, and Pietro Ferraro, Reversible Fragmentation and Self-Assembling of Nematic Liquid Crystal Droplets on Functionalized Pyroelectric Substrates, Adv. Funct. Mater., 22, 32673272 (2012).

[13] J. L. Carns, G. Cook, M. A. Saleh, S. V. Serak, N. Tabiryan, and D. R. Evans, Self-activated liquid-crystal cells with photovoltaic substrates, Opt. Lett. 31, 993 (2006).

[14] S.-T. Wu, U. Efron, and L. D. Hess, Birefringence measurement of liquid crystals, Appl. Opt. 23, 3912 (1984).

[15] L. Lucchetti, K. Kushnir, F. Ciciulla, A. Zaltron, G. Bettella, G. Pozza, C. Sada, V. Reshetnyak, F. Simoni, All-optical phase shifter with photovoltaic liquid crystal cell, Proceedings of SPIE vol. 9940, 99400G1 (2016).

[16] K. Buse, J. Imbrock, E. Kratzig and K. Peithmann, Photorefractive Effects in $\mathrm{LiNbO}_{3}$ and $\mathrm{LiTaO}_{3}$, in Photorefractive Materials and their applications 2, P. Gunter, J.P. Huignard, Springer (2007).

[17] K. Buse, Light-induced charge transport processes in photorefractive crystals I: models and experimental methods, Appl. Phys. B 64, 273-291 (1997).

[18] F. Simoni, Nonlinear Optical Properties of Liquid Crystals and Polymer Dispersed Liquid Crystals (World Scientific, 1997).

[19] Javier Villarroel, Héctor Burgos, Ángel García-Cabañes, Mercedes Carrascosa, Alfonso Blázquez-Castro, and Fernando Agulló-López, Photovoltaic versus optical tweezers, Opt. Express, 19, 24320-24330 (2011).

[20] C. Braun, S. Sanna, and W. G. Schmidt, Liquid Crystal (8CB) Molecular Adsorption on Lithium Niobate Z-Cut Surfaces, J. Phys. Chem. C, 119, 9342-9346 (2015).

[21] S. M. Kostritskii, M. Aillerie and O. G. Sevostyanov, Selfcompensation of optical damage in reduced nominally pure $\mathrm{LiNbO}_{3}$ crystals, J. Appl. Phys. 107, 123526 (2010).

[22] Igor Musevic, Miha Skarabot, Uros Tkalec, Miha Ravnik, Slobodan Zumer, Two-Dimensional Nematic

Colloidal Crystals Self-Assembled by Topological Defects, Science. 313, 954 (2006).

[23] U. Tkalec, M. Ravnik, S. Copar, S. Zumer, I. Musevic, Reconfigurable knots and links in chiral nematic colloids, Science 333, 62 (2011).

[24] E. Brasselet, N. Murazawa, H. Misawa, S. Juodkazis, Optical Vortices from Liquid Crystal Droplets, Phys. Rev. Lett. 103, 103903 (2009).

[25] E. Brasselet, Spin-orbit optical cross-phase-modulation, Phys. Rev. A. 82, 063836 (2010).

[26] L. Criante, F. Bracalente, L. Lucchetti, F. Simoni, E. Brasselet, Electrically tunable optoelastic interaction range of nematic colloids, Soft Matter. 9, 5459 (2013).

[27] M. Esseling, A. Zaltron, C. Sada and C. Denz, Charge sensor and particle trap based on z-cut lithium niobate, Appl. Phys. Lett., 103, 061115 (2013). 